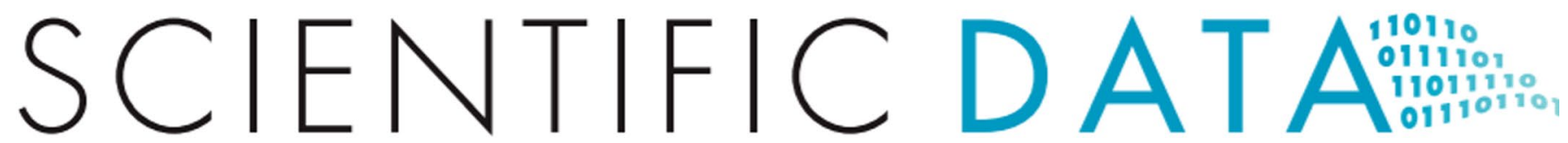

OPEN

Published online: 19 March 2019

\title{
Addendum: The FAIR Guiding Principles for scientific data management and stewardship
}

Mark D. Wilkinson et al.\#

Addendum to: Scientific Data https://doi.org/10.1038/sdata.2016.18, published online 15 March 2016

Since publication, the URL at which the FAIR principles 'living document' is hosted and maintained has changed from http://datafairport.org/fair-principles-living-document-menu to https://www.go-fair.org/fair-principles/.

(c) (i) Open Access This article is licensed under a Creative Commons Attribution 4.0 International License, which permits use, sharing, adaptation, distribution and reproduction in any medium or format, as long as you give appropriate credit to the original author(s) and the source, provide a link to the Creative Commons license, and indicate if changes were made. The images or other third party material in this article are included in the article's Creative Commons license, unless indicated otherwise in a credit line to the material. If material is not included in the article's Creative Commons license and your intended use is not permitted by statutory regulation or exceeds the permitted use, you will need to obtain permission directly from the copyright holder. To view a copy of this license, visit http://creativecommons.org/licenses/by/4.0/.

(c) The Author(s) 2019 
Mark D. Wilkinson ${ }^{1}$, Michel Dumontier ${ }^{2}$, ljsbrand Jan Aalbersberg ${ }^{3}$, Gabrielle Appleton ${ }^{3}$, Myles Axton ${ }^{4}$, Arie Baak ${ }^{5}$, Niklas Blomberg 6 , Jan-Willem Boiten ${ }^{7}$, Luiz Bonino da Silva Santos ${ }^{8}$, Philip E. Bourne ${ }^{9}$, Jildau Bouwman ${ }^{10}$, Anthony J. Brookes ${ }^{11}$, Tim Clark $^{12}$, Mercè Crosas ${ }^{13}$, Ingrid Dillo ${ }^{14}$, Olivier Dumon ${ }^{3}$, Scott Edmunds ${ }^{15}$, Chris T. Evelo ${ }^{16}$, Richard Finkers ${ }^{17}$, Alejandra Gonzalez-Bel$\operatorname{tran}^{18}$, Alasdair J. G. Gray ${ }^{19}$, Paul Groth ${ }^{3}$, Carole Goble ${ }^{20}$, Jeffrey S. Grethe ${ }^{21}$, Jaap Heringa ${ }^{22}$, Peter A. C. 't Hoen ${ }^{23}$, Rob Hooft ${ }^{24}$, Tobias Kuhn' ${ }^{25}$, Ruben Kok ${ }^{22}$, Joost Kok ${ }^{26}$, Scott J. Lusher ${ }^{27}$, Maryann E. Martone ${ }^{28}$, Albert Mons ${ }^{29}$, Abel L. Packer ${ }^{30}$, Bengt Persson ${ }^{31}$, Philippe Rocca-Serra ${ }^{18}$, Marco Roos ${ }^{32}$, Rene van Schaik ${ }^{33}$, Susanna-Assunta Sansone ${ }^{18}$, Erik Schultes ${ }^{34}$, Thierry Sengstag ${ }^{35}$, Ted Slater ${ }^{36}$, George Strawn ${ }^{37}$, Morris A. Swertz ${ }^{38}$, Mark Thompson ${ }^{32}$, Johan van der Lei ${ }^{39}$, Erik van Mulligen ${ }^{39}$, Jan Velterop ${ }^{40}$, Andra Waagmeester ${ }^{41}$, Peter Wittenburg ${ }^{42}$, Katherine Wolstencroft ${ }^{43}$, Jun Zhao ${ }^{44}$ \& Barend Mons ${ }^{45,46,47}$

${ }^{1}$ Center for Plant Biotechnology and Genomics, Universidad Politécnica de Madrid, Madrid, 28223, Spain. ${ }^{2}$ Stanford University, Stanford, 94305-5411, USA. ${ }^{3}$ Elsevier, Amsterdam, 1043 NX, The Netherlands. ${ }^{4}$ Nature Genetics, New York, 10004-1562, USA. ${ }^{5}$ Euretos and Phortos Consultants, Rotterdam, 2741 CA, The Netherlands. ${ }^{6}$ ELIXIR, Wellcome Genome Campus, Hinxton, CB10 1SA, UK. ${ }^{7}$ Lygature, Eindhoven, 5656 AG, The Netherlands. ${ }^{8}$ Vrije Universiteit Amsterdam, Dutch Techcenter for Life Sciences, Amsterdam, $1081 \mathrm{HV}$, The Netherlands. ${ }^{9}$ Office of the Director, National Institutes of Health, Rockville, 20892, USA. ${ }^{10} \mathrm{TNO}$, Zeist, $3700 \mathrm{AJ}$, The Netherlands. ${ }^{11}$ Department of Genetics, University of Leicester, Leicester, LE1 7RH, UK. ${ }^{12}$ Harvard Medical School, Boston, Massachusetts, MA, 02115, USA. ${ }^{13}$ Harvard University, Cambridge, Massachusetts, MA, 02138, USA. ${ }^{14}$ Data Archiving and Networked Services (DANS), The Hague, $2593 \mathrm{HW}$, The Netherlands. ${ }^{15} \mathrm{GigaScience,} \mathrm{Beijing} \mathrm{Genomics} \mathrm{Institute,} \mathrm{Shenzhen,}$ 518083, China. ${ }^{16}$ Department of Bioinformatics, Maastricht University, Maastricht, 6200 MD, The Netherlands. ${ }^{17}$ Wageningen UR Plant Breeding, Wageningen, 6708 PB, The Netherlands. ${ }^{18}$ Oxford e-Research Center, University of Oxford, Oxford, OX1 3QG, UK. ${ }^{19}$ Heriot-Watt University, Edinburgh, EH14 4AS, UK. ${ }^{20}$ School of Computer Science, University of Manchester, Manchester, M13 9PL, UK. ${ }^{21}$ Center for Research in Biological Systems, School of Medicine, University of California San Diego, La Jolla, California, 92093-0446, USA. ${ }^{22}$ Dutch Techcenter for the Life Sciences, Utrecht, 3501 DE, The Netherlands. ${ }^{23}$ Department of Human Genetics, Leiden University Medical Center, Dutch Techcenter for the Life Sciences, Leiden, 2300 RC, The Netherlands. ${ }^{24}$ Dutch TechCenter for Life Sciences and ELIXIR-NL, Utrecht, 3501 DE, The Netherlands. ${ }^{25}$ VU University Amsterdam, Amsterdam, 1081 HV, The Netherlands. ${ }^{26}$ Leiden Center of Data Science, Leiden University, Leiden, 2300 RA, The Netherlands. ${ }^{27}$ Netherlands eScience Center, Amsterdam, 1098 XG, The Netherlands. ${ }^{28}$ National Center for Microscopy and Imaging Research, UCSD, San Diego, 92103, USA. ${ }^{29}$ Phortos Consultants, San Diego, 92011, USA. ${ }^{30}$ SciELO/FAPESP Program, UNIFESP Foundation, São Paulo, 05468-901, Brazil. ${ }^{31}$ Bioinformatics Infrastructure for Life Sciences (BILS), Science for Life Laboratory, Dept of Cell and Molecular Biology, Uppsala University, S-751 24, Uppsala, Sweden. ${ }^{32}$ Leiden University Medical Center, Leiden, 2333 ZA, The Netherlands. ${ }^{33}$ Bayer CropScience, Gent Area, 1831, Belgium. ${ }^{34}$ Leiden Institute for Advanced Computer Science, Leiden University Medical Center, Leiden, 2300 RA, The Netherlands. ${ }^{35}$ Swiss Institute of Bioinformatics and University of Basel, Basel, 4056, Switzerland. ${ }^{36}$ Cray, Inc., Seattle, 98164, USA. ${ }^{37}$ Unaffiliated, Washington, D.C., USA. ${ }^{38}$ University Medical Center Groningen (UMCG), University of Groningen, Groningen, $9713 \mathrm{GZ}$, The Netherlands. ${ }^{39}$ Erasmus MC, Rotterdam, 3015 CE, The Netherlands. ${ }^{40}$ Independent Open Access and Open Science Advocate, Guildford, GU1 3PW, UK. ${ }^{41}$ Micelio, Antwerp, 2180, Belgium. ${ }^{42}$ Max Planck Compute and Data Facility, MPS, Garching, 85748, Germany. ${ }^{43}$ Leiden Institute of Advanced Computer Science, Leiden University, Leiden, 2333 CA, The Netherlands. ${ }^{44}$ Department of Computer Science, Oxford University, Oxford, OX1 30D, UK. ${ }^{45}$ Leiden University Medical Center, Leiden and Dutch TechCenter for Life Sciences, Utrecht, 2333 ZA, The Netherlands. ${ }^{46}$ Netherlands eScience Center, Amsterdam, 1098XG, The Netherlands. ${ }^{47}$ Erasmus MC, Rotterdam, 3015 CE, The Netherlands. 